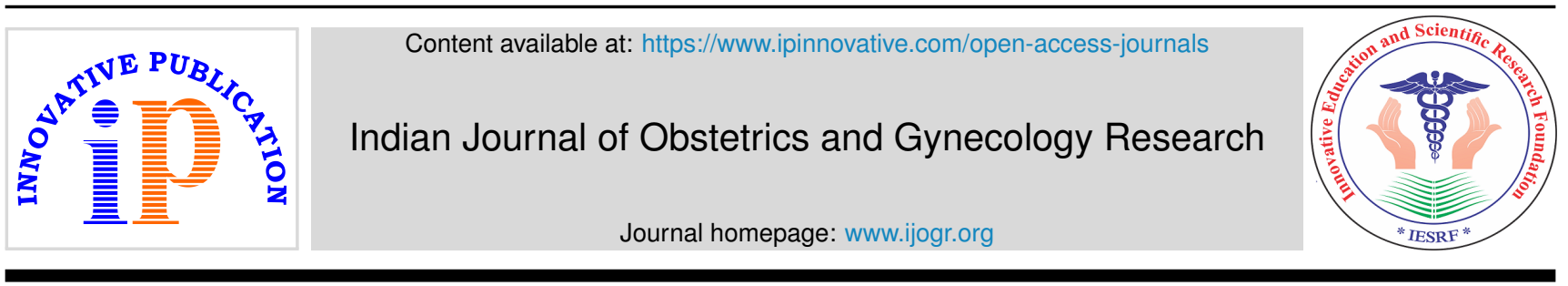

Original Research Article

\title{
Prevalence of anti-thyroid peroxidase antibodies in the first trimester pregnancy and its impact on maternal and fetal outcomes in a tertiary care centre in Bangalore
}

\author{
Shruthi H S ${ }^{1}$, , Nalini Arunkumar ${ }^{1}$, Ravi N Patil ${ }^{1}$ \\ ${ }^{1}$ Dept. of Obstetrics and Gynaecology, Bangalore Baptist Hospital, Bengaluru, Karnataka, India
}

\section{A R T I C L E I N F O}

Article history:

Received 14-01-2021

Accepted 12-02-2021

Available online 11-06-2021

Keywords:

Antenatal complications

Anti TPO Ab

Hypothyroidism

\begin{abstract}
A B S T R A C T
Background: Hypothyroidism during pregnancy has an adverse effect on both mother and child. The maternal and foetal risk is higher in TPOAb (Thyroid peroxidase antibody) positive women compared to women with negative TPO Ab. The recent ATA (American Thyroid Association) guidelines recommend that pregnant women with TSH (Thyroid Stimulating Hormone) concentration above $2.5 \mathrm{mU} / \mathrm{L}$ should be evaluated for TPOAb status and LT4(levothyroxine) treatment should be considered with TSH values between $2.5 \mathrm{mU} / \mathrm{L}$ and $4.0 \mathrm{mU} / \mathrm{L}$ only when TPOAb status is positive.

Materials and Methods: All the pregnant women booked in first trimester underwent testing for TSH levels and subsequently for anti TPO Ab if TSH levels were between 2.5-4 $\mathrm{mIU} / \mathrm{ml}$. The hospital based prevalence of women with anti TPO Ab positive status was determined. These pregnancies were followed till term and the maternal and foetal complications associated with TPO Ab positive and negative status were compared.

Results: Total of 400 pregnant women were included. The hospital prevalence of women with anti TPO antibodies in first trimester of pregnancy with TSH values between $2.5-4 \mathrm{mIU} / \mathrm{ml}$ was found to be 23.5\%. Anti TPO antibody positive status was significantly more associated with antenatal complications especially GDM and IUGR as compared to patients with anti TPO antibody negative status $(47.8 \% \mathrm{v} / \mathrm{s}$ $23.2 \%$, p value 0.001 ).

Conclusion: Women with TPO Ab positive status are to be vigilantly monitored for early detection and management of various antenatal complications. Determining anti TPO Ab status helps in avoiding unnecessary treatment of the women with TPO Ab negative status and TSH between $2.5-4 \mathrm{mIU} / \mathrm{ml}$.

(C) This is an open access article distributed under the terms of the Creative Commons Attribution License (https://creativecommons.org/licenses/by/4.0/) which permits unrestricted use, distribution, and reproduction in any medium, provided the original author and source are credited.
\end{abstract}

\section{Introduction}

Thyroid disorders are common in young women and thus frequently encountered during pregnancy. The prevalence of thyroid dysfunction during pregnancy is estimated to be $2-3 \% .^{1}$ Hypothyroidism during pregnancy has an adverse effect on both the mother and the child. Hypothyroidism has been found to be associated with pregnancy complications such as hypertension, preterm birth, low birth weight, placental abruption, and fetal death, fetal growth restrictions, fetal thyroid dysfunction, fetal

\footnotetext{
* Corresponding author.

E-mail address: shruthihs910@gmail.com (Shruthi H S).
}

neuro developmental dysfunction. ${ }^{2}$

Autoimmune thyroid disease (AITD) leads to adverse outcomes in pregnancy by causing progressive thyroid dysfunction. Thyroid autoimmunity may also be associated with a higher frequency of preterm deliveries and abruption placenta. Data from the recently published studies emphasises the association between miscarriage and preterm delivery in women with normal thyroid function but test positive for thyroid peroxidase antibodies. ${ }^{3}$ During first trimester, approximately one in ten pregnant women develop antibodies to TPO or thyroglobulin and hypothyroidism develops in roughly $16 \%$ of these women. ${ }^{4}$ 
A study has stratified the risk imparted by hypothyroidism according to TPOAb status and consistently show that this risk is higher in TPOAb positive women even when the thyroid function is identical. ${ }^{5}$ Prompt identification of thyroid disorder and timely initiation of therapy in pregnancy is essential. The trimester specific normal ranges for TSH were as follows- First trimester: $0.1-2.5 \mathrm{mIU} / \mathrm{ml}$, Second and third trimester: 0.2-3.0 $\mathrm{mIU} / \mathrm{ml}$.

The American Thyroid Association (ATA) thyroid and pregnancy guidelines 2017 has modified the pregnancy specific TSH reference range as follows- If internal or transferable pregnancy specific TSH reference ranges are not available, an upper reference limit of $\sim 4.0 \mathrm{mU} / \mathrm{L}$ maybe used. $^{5}$

It also recommends that all pregnant women with TSH concentration above $2.5 \mathrm{mU} / \mathrm{L}$ should be evaluated for TPOAb status and that LT4(Levothyroxine) treatment should be considered for euthyroid pregnant women with TPOAb positive status with TSH status between $2.5 \mathrm{mU} / \mathrm{L}$ and $4.0 \mathrm{mU} / \mathrm{L}^{5}$

\section{Materials and Methods}

This prospective observational study was conducted in Department of Obstetrics and Gynecology, in our tertiary care hospital from October 2017 to December 2019.

The primary objective of our study was to determine the TPO $\mathrm{Ab}$ status of pregnant women in their first trimester of pregnancy with TSH levels between 2.5$4 \mathrm{mIU} / \mathrm{ml}$. The secondary objective was to compare the maternal and fetal complications associated with TPO Ab positive and negative status in pregnant women such as early pregnancy loss, pre-eclampsia, gestational diabetes mellitus, intrauterine growth restriction, intrauterine fetal death.

All pregnant women in first trimester of pregnancy with TSH values between $2.5-4 \mathrm{mIU} / \mathrm{ml}$ were included after obtaining consent. Pregnant women with hypothyroidism preconceptionally were excluded from the study. Total 400 pregnant women were included in the study. Subjects underwent testing to determine the anti TPO Ab status and were treated according to the ATA guidelines 2017. The pregnancy was followed till term. Thus, the hospital based prevalence of the anti TPO Ab was determined and the maternal and foetal complications associated with TPO Ab positive and negative status were compared.

\subsection{Statistical analysis}

The data collected was entered into an Excel sheet. The hospital-based prevalence was calculated. The maternal and foetal outcomes with Anti TPO Ab positive and negative status were compared. For continuous variables, differences between the groups were analysed by the Chi square test and $p<0.05$ was considered significant. Logistic regression was done to adjust for the potential confounders and also to determine the antenatal complications associated with anti TPO antibodies. $\mathrm{p}$ value $<0.005$ was considered significant.

\section{Results}

\subsection{Characteristics of study population}

A total of 400 women in first trimester of pregnancy with TSH between $2.5-4 \mathrm{mIU} / \mathrm{ml}$ were recruited in the study. Majority of the women were in the age group of 26-30 years. Primigravid and multigravid women were equally distributed in the study population. About $49 \%$ of the pregnant women had normal pre pregnancy BMI and most of them did not have any medical or surgical comorbidities prior to conception. Eight percent of the included women gave family history of hypothyroidism (among parents).

\subsection{Antenatal complications}

Twenty nine percent of the total women recruited developed antenatal complications such as GDM, hypertensive disorders, IUGR, early pregnancy loss, IUD, oligohydramnios. Among these, GDM, hypertensive disorders and IUGR were found to be more in number compared to the other complications.

\subsection{Presence of anti TPO Ab}

Ninety-four women recruited in the study tested positive for anti TPO Ab, and hence the hospital-based prevalence of anti TPO Ab was calculated to be $23.5 \%$.

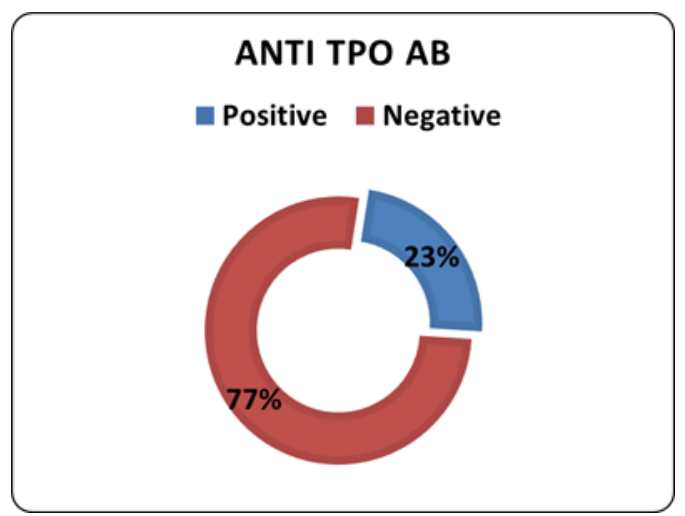

Fig. 1: Hospital based prevalence of anti TPOAb

\subsection{Association with anti TPO Ab}

Analysis was done to note if the various parameters in the study population were significantly associated with Anti TPO Ab positivity. Among the women positive for TPO Ab, $17 \%$ had history of hypothyroidism in their parents. Fifty percent of those who had family history of hypothyroidism tested positive for TPO Ab. This was found to be significant 
Table 1: Baseline characteristics of the women

\begin{tabular}{|c|c|c|c|}
\hline \multirow{2}{*}{ Parameters } & \multirow{2}{*}{ Overall $(\mathrm{N}=\mathbf{4 0 0})$} & \multicolumn{2}{|c|}{ Anti TPO AB } \\
\hline & & Negative $(\mathrm{N}=306)$ & Positive ( $N=94)$ \\
\hline \multicolumn{4}{|l|}{ Age } \\
\hline$<=25$ Years & $152(38 \%)$ & $113(36.9 \%)$ & $39(41.5 \%)$ \\
\hline 26-30 Years & $166(41.5 \%)$ & $129(42.2 \%)$ & $37(39.4 \%)$ \\
\hline$>30$ Years & $82(20.5 \%)$ & $64(20.9 \%)$ & $18(19.1 \%)$ \\
\hline \multicolumn{4}{|l|}{ BMI } \\
\hline Underweight & $11(2.8 \%)$ & $9(2.9 \%)$ & $2(2.1 \%)$ \\
\hline Normal & $195(48.8 \%)$ & $143(46.7 \%)$ & $52(55.3 \%)$ \\
\hline Overweight & $113(28.3 \%)$ & $88(28.8 \%)$ & $25(26.6 \%)$ \\
\hline Obese & $81(20.3 \%)$ & $66(21.6 \%)$ & $15(16 \%)$ \\
\hline \multicolumn{4}{|l|}{ Gravida } \\
\hline Primi & $210(52.5 \%)$ & $157(51.3 \%)$ & $53(56.4 \%)$ \\
\hline Multi & $190(47.5 \%)$ & $149(48.7 \%)$ & $41(43.6 \%)$ \\
\hline \multicolumn{4}{|l|}{ Comorbidities } \\
\hline Present & $30(7.5 \%)$ & $21(6.9 \%)$ & $9(9.6 \%)$ \\
\hline Absent & $370(92.5 \%)$ & $285(93.1 \%)$ & $85(90.4 \%)$ \\
\hline \multicolumn{4}{|c|}{ Family H/O Hypothyroidism } \\
\hline Present & $32(8 \%)$ & $16(5.2 \%)$ & $16(17 \%)$ \\
\hline Absent & $368(92 \%)$ & $290(94.8 \%)$ & $78(83 \%)$ \\
\hline \multicolumn{4}{|l|}{ GDM } \\
\hline Present & $53(13.3 \%)$ & $32(10.5 \%)$ & $21(22.3 \%)$ \\
\hline Absent & $347(86.8 \%)$ & $274(89.5 \%)$ & $73(77.7 \%)$ \\
\hline \multicolumn{4}{|l|}{ HTN Disorder } \\
\hline Present & $25(6.3 \%)$ & $16(5.2 \%)$ & $9(9.6 \%)$ \\
\hline Absent & $375(93.8 \%)$ & $290(94.8 \%)$ & $85(90.4 \%)$ \\
\hline \multicolumn{4}{|l|}{ Mode of delivery $\S$} \\
\hline Vaginal delivery & $203(66.6 \%)$ & $151(66.5 \%)$ & $52(66.7 \%)$ \\
\hline LSCS & $102(33.4 \%)$ & $76(33.5 \%)$ & $26(33.3 \%)$ \\
\hline \multicolumn{4}{|l|}{ IUGR } \\
\hline Present & $11(2.8 \%)$ & $4(1.3 \%)$ & $7(7.4 \%)$ \\
\hline Absent & $389(97.3 \%)$ & $302(98.7 \%)$ & $87(92.6 \%)$ \\
\hline
\end{tabular}

Percentages aregiven in parenthesis

Table 2: Antenatal complications among study group

\begin{tabular}{lc}
\hline ANC Complication & No of women affected \\
GDM & 53 \\
Hypertensive disorders in pregnancy & 25 \\
IUGR and Oligohydramnios & 29 \\
Preterm delivery & 1 \\
IUD & 4 \\
Early pregnancy loss & 3 \\
Abruptio placenta & 4
\end{tabular}

( $\mathrm{p}<0.001$ ). The Anti TPO Ab positive status and the development of complications in the antenatal period were found to be significantly associated with a $\mathrm{p}$ value of $<0.001$.

None of the other parameters such as age, parity, BMI, mode of delivery was found to be significantly associated with anti TPO positivity.

On multivariate analysis, development of GDM and IUGR had a significant $\mathrm{p}$ value for the association with anti TPO Ab ( $\mathrm{p}=0.004$ and 0.003 respectively) as shown in Table 3.

\section{Discussion}

The study is a prospective observational study in which 400 consenting women were recruited over the study periodfrom October $1^{\text {st }} 2017$ to December $31^{\text {st }}$ 2019. Each one of them underwent TSH testing as a part of their routine antenatal checkup. Those women with TSH values between 2.5 to $4 \mathrm{mIU} / \mathrm{ml}$ underwent testing for anti TPO Ab as per the ATA 2017 guidelines and were treated accordingly.

Primary analysis of the data collected from the study samples showed the hospital-based prevalence of anti TPO 
Table 3: Univariate \& multivariate logistic regression analysis

\begin{tabular}{|c|c|c|c|c|}
\hline \multirow{2}{*}{ Parameters } & \multicolumn{2}{|c|}{ Univariate } & \multicolumn{2}{|c|}{ Multivariate } \\
\hline & OR $(95 \%$ C.I $)$ & P-Value & AOR (95\% C.I) & P-Value \\
\hline Age & & & - & - \\
\hline$<=25$ Years & REF & & - & - \\
\hline 26-30 Years & $0.83(0.5,1.39)$ & 0.482 & - & - \\
\hline$>30$ Years & $0.81(0.43,1.54)$ & 0.529 & - & - \\
\hline BMI & & & - & - \\
\hline Underweight & $0.61(0.13,2.92)$ & 0.537 & - & - \\
\hline Normal & REF & & - & - \\
\hline Overweight & $0.78(0.45,1.35)$ & 0.375 & - & - \\
\hline Obese & $0.63(0.33,1.19)$ & 0.153 & - & \\
\hline Gravida & & & & - \\
\hline Primi & REF & - & - & - \\
\hline Multi & $0.82(0.51,1.3)$ & 0.389 & - & - \\
\hline Comorbidities & & & - & - \\
\hline Present & $1.44(0.63,3.25)$ & 0.385 & - & - \\
\hline Absent & REF & & - & - \\
\hline \multicolumn{5}{|l|}{$\begin{array}{l}\text { Family h/o } \\
\text { hypothyroidism }\end{array}$} \\
\hline Present & $3.72(1.78,7.77)$ & $<0.001$ & $3.71(1.74,7.88)$ & 0.001 \\
\hline Absent & REF & & REF & \\
\hline \multicolumn{5}{|l|}{ IUGR } \\
\hline Present & $6.07(1.74,21.23)$ & 0.005 & $6.81(1.9,24.37)$ & 0.003 \\
\hline Absent & REF & & REF & \\
\hline \multicolumn{5}{|l|}{ GDM } \\
\hline Present & $2.46(1.34,4.52)$ & 0.004 & $2.51(1.34,4.69)$ & 0.004 \\
\hline Absent & REF & & REF & \\
\hline \multicolumn{5}{|l|}{ Htn disorder } \\
\hline Present & $1.92(0.82,4.5)$ & 0.134 & & \\
\hline Absent & REF & & & \\
\hline \multicolumn{5}{|l|}{ Mode of delivery } \\
\hline Vaginal delivery & REF & & & \\
\hline LSCS & $0.99(0.58,1.71)$ & 0.981 & & \\
\hline
\end{tabular}

OR - Odds Ratio; AOR - Adjusted Odds Ratio; C.I - Confidence Interval

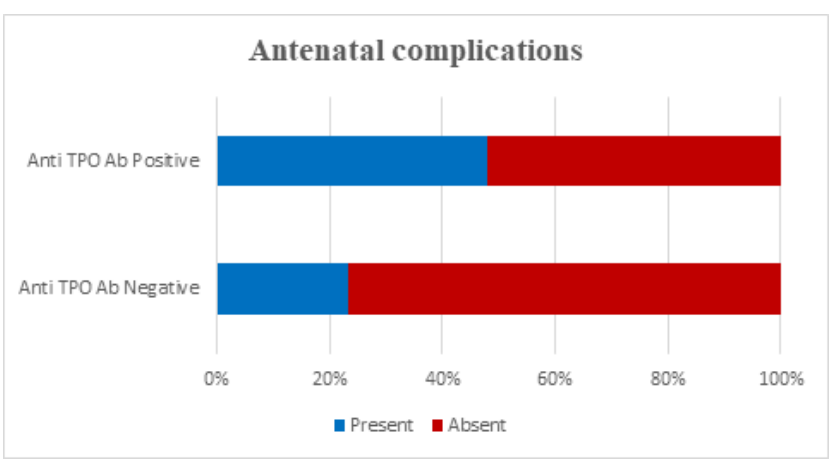

Fig. 2: Presence of anti TPO Ab and development of antenatal complications

antibodies in first trimester of pregnancy with TSH values between $2.5-4 \mathrm{mIU} / \mathrm{ml}$ as $23.5 \%$.

The extended analysis of the data obtained showed that anti TPO antibody positive status was significantly more associated with antenatal complications such as GDM, hypertensive disorders, IUGR, IUD, early pregnancy loss, oligohydramnios as compared to patients with anti TPO antibody negative status. Out of the patients with TPO antibody status, $47.8 \%$ developed antenatal complications at follow up, whereas only $23.2 \%$ of the patients with TPO antibody negative status developed antenatal complications. This difference is statistically significant as evident by a $p$ value of $<0.001$. Each of the various antenatal complications seen were separately analyzed and GDM and IUGR were found to be significantly associated with the anti TPO antibodies. Though in general, antenatal complications are said to be more in women with anti TPO $\mathrm{Ab}$ positivity, various studies have shown association of different complications with TPO Ab. Few studies have shown association of anti TPO Ab with increased risk of miscarriages and preterm delivery, ${ }^{6}$ infertility and anemia, ${ }^{7}$ low birth weight. ${ }^{8}$ It is also interesting to note that the Anti TPO Ab were significantly in higher number in the group of women who had family history of hypothyroidism (in either 
Table 4: Comparison of various studies

\begin{tabular}{llcc}
\hline Year & Study by & Prevalence of TPO Ab & $\begin{array}{c}\text { Association between TPO Ab positivity } \\
\text { and antenatal complications }\end{array}$ \\
2015 & Rajesh Rajput $^{3}$ & $27.8 \%$ & - \\
2016 & Monika Meena $^{7}$ & $11 \%$ & Yes \\
2016 & Aruna Meena $^{8}$ & $6 \%$ & Yes \\
2017 & Rajesh Rajput $^{6}$ & $18.9 \%$ & Yes \\
2019 & Our study & $23.5 \%$ & Yes \\
\hline
\end{tabular}

of the parents).

Previously the cut off value for TSH in the first trimester of pregnancy was $2.5 \mathrm{mIU} / \mathrm{ml}$. This was revised to $4 \mathrm{mIU} / \mathrm{ml}$ in the updated ATA 2017 guidelines. Also, the guidelines recommended that those pregnant women with TSH values more than $2.5 \mathrm{mIU} / \mathrm{ml}$ should undergo testing for anti TPO $\mathrm{Ab}$. Guidelines say that the women are to be provided with thyroid hormone replacement only if she tests positive for anti TPO antibodies when the TSH is between 2.5$4 \mathrm{mIU} / \mathrm{ml}$. This is due to fact that patients with TPO $\mathrm{Ab}$ positive status tend to develop more antenatal complications as compared to women with negative status with the same TSH value. ${ }^{6}$ This was established in our study as women with TPO positivity had more complications compared to the those who were negative.

Many studies have been done internationally and in India to identify the prevalence of the anti TPO Ab positivity among the local population and its association between the antenatal complications. The results obtained in this study are comparable to the others in means of prevalence and the associated complications. This has been summarized in the Table 4.

It is important to note that the prevalence of anti TPO $\mathrm{Ab}$ when TSH levels are between $2.5-4 \mathrm{mIU} / \mathrm{ml}$ is quite low, that is $23.5 \%$. It implies that $76.5 \%$ of the pregnant women with TSH values between $2.5-4 \mathrm{mIU} / \mathrm{ml}$ need not take any thyroid replacement therapy in the form of Levothyroxine tablets. Though the cost of anti TPO Ab testing is high (about Rs1300 in our study setting), it can prove beneficial in the regard that the financial expenses of taking medications for thyroid replacement throughout pregnancy and in the postpartum period is lowered. About $77 \%$ of the cases undergoing unnecessary treatment with thyroid replacement can be avoided by using this small test for detection of the anti TPO Ab.

The secondary analysis has also shown that women with anti TPO Ab are more prone to develop antenatal complications, especially GDM and IUGR.

Thus, it is implied that those women who test positive for anti TPO Ab need more vigilant monitoring and the fact that they are more prone for antenatal complications must be considered throughout their pregnancy.

The fact that the women with anti TPO Ab developed significantly more antenatal complications even after the treatment with levothyroxine remains debatable. Most of the available studies on anti TPO $\mathrm{Ab}$ in pregnancy and the maternal and fetal outcomes are based on women with euthyroid status where they were not treated with levothyroxine. ${ }^{6-9}$ Whether an increased dose of levothyroxine or any other additional treatment is required in hypothyroid pregnant women with anti TPO Ab positive status is to be investigated by large studies.

\section{Limitations}

1. Small sample size- Recruiting a larger sample size would bring out a more accurate prevalence of the anti TPO $\mathrm{Ab}$ in the local population and stronger association between the TPO Ab positivity and antenatal complications.

2. Further studies are required to establish a population based upper limit for the TSH in each trimester of pregnancy as recommended by the ATA guidelines 2017.

\section{Conclusions}

The results showed that the hospital-based prevalence of anti TPO Ab positivity to be $23.5 \%$. The study also showed that anti TPO Ab positivity is significantly associated with various antenatal complications, especially GDM and IUGR, as evident by a statistically significant $\mathrm{p}$ value. Among those who tested positive for anti TPO Ab, 47.8\% developed various antenatal complications, whereas only $23.2 \%$ developed complications among those who tested negative. The advantage of performing the anti TPO Ab test in the pregnant women is that more than $75 \%$ of the women with TSH between $2.5-4 \mathrm{mIU} / \mathrm{ml}$ can avoid thyroxine replacement therapy as they are going to test negative for anti TPO Ab.

\section{Recommendations}

All women with TSH more than $2.5 \mathrm{mIU} / \mathrm{ml}$ in first trimester of pregnancy should undergo testing for anti TPO $\mathrm{Ab}$. Women who test positive for anti TPO Ab must be closely monitored during pregnancy as they are more prone for various antenatal complications compared to those who test negative. Women with TSH between 2.5 to $4 \mathrm{mIU} / \mathrm{ml}$ in first trimester of pregnancy are to be treated only if the anti $\mathrm{TPO} \mathrm{Ab}$ is positive. 


\section{Source of Funding}

None.

\section{Conflict of Interest}

The authors declare that there is no conflict of interest.

\section{References}

1. Azizi F, Delshad H. Thyroid Derangements in Pregnancy. Iran $J$ Endocrinol Metab. 2014;15:491-508.

2. Nazarpour S, Ramezani F. Masoumeh Simbar et al;Thyroid dysfunctions and pregnancy outcomes. Iran $J$ Reprod Med. 2015;13(7):387-96.

3. Rajput R, Goel V, Nanda S, Rajput M, Seth S. Prevalence of thyroid dysfunction among women during the first trimester of pregnancy at a tertiary care hospital in Haryana. Indian J Endocrinol Metab. 2015;19(3):416-9. 10:-10.4103/2230-8210.52/पा

4. Dhanwal DK, Bajaj S, Rajput R, Subramaniam KAV, Chowdhury $\mathrm{S}$, Bhandari R, et al. Prevalence of hypothyroidism in pregnancy: An epidemiological study from 11 cities in 9 states of India. Indian J Endocrinol Metab. 2016;20(3):387-90. 101:10.4103/2230-

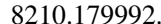

5. Alexander EK, Pearce EN, Brent GA, Brown RS, Chen H, Dosiou C, et al. 2017 Guidelines of the American Thyroid Association for the Diagnosis and Management of Thyroid Disease During Pregnancy and the Postpartum. Thyroid. 2017;27(3):315-89. doi:10.1089/thy.2016.0457.

6. Rajput R, Yadav T, Seth S, Nanda S. Prevalence of thyroid peroxidase antibody and pregnancy outcome in euthyroid autoimmune positive pregnant women from a tertiary care center in Haryana. Indian J Endocrinol Metab. 2017;21(4):577-80. doi:10.4103/1jem.ijem_397_16.

7. Meena M, Chopra S, Jain V, Aggarwal N. The Effect of Anti-Thyroid Peroxidase Antibodies on Pregnancy Outcomes in Euthyroid Women. $J$ Clin Diagn Res. 2016;10(9):4-07.

8. Meena A, Nagar P. Pregnancy Outcome in Euthyroid Women with Anti-Thyroid Peroxidase Antibodies. J Obstet Gynecol India 2016;66(3):160-5. 100:101007/s[3224-014-0657-6.

9. Yuan N, Sun J, Li Z, Chai S, Zhang X, Ji L. Relationship between anti thyroid peroxidase antibody positivity and pregnancy- related and fetal outcomes in euthyroid women: a single- center cohort study. BMC Pregnancy Childbirth. 2020;20(1):491.

\section{Author biography}

Shruthi H S, PG Resident

Nalini Arunkumar, HOD

Ravi N Patil, Senior Consultant

Cite this article: Shruthi H S, Arunkumar N, Patil RN. Prevalence of anti-thyroid peroxidase antibodies in the first trimester pregnancy and its impact on maternal and fetal outcomes in a tertiary care centre in Bangalore. Indian J Obstet Gynecol Res 2021;8(2):182-187. 